\title{
Sprachkurs rund ums Sterbezimmer
}

\author{
Jann P. Schwarzenbach \\ Dr. med., Facharzt für Allgemeinmedizin, Mitglied FMH
}

Die Problematik des Sterbens bringt uns natürlicherweise dazu, über das Wesen und die Sinnhaftigkeit der Welt und unseres eigenen Lebens nachzudenken. Und da wird auch manch nüchterner Mensch auf klassische religiöse und philosophische Themen stossen, unter anderem auf den Dauerbrenner unseres zeitlich limitierten Daseins. Für die Idee eines Fortbestehens von körperlichen Strukturen mag sich heutzutage, schon aus Platzgründen, zwar kaum mehr jemand stark machen, und auch mit den raumsparenden seelischen Elementen, wie der ständigen Selbsterfahrung, unserem liebgewonnenen empirischen Ich, wird es wohl nach dem endgültigen Abschalten der neuralen Schaltkreise vorbei sein. Unter Ärzten und Ärztinnen gesagt, hat diese Einsicht auch ihr Gutes, welcher Kollege möchte schon mit allen lieben "Chronophagen» vom Wartezimmer und Behandlungsraum die eigene Ewigkeit teilen! Dessen ungeachtet, schmecken die leicht verdaulichen, rein materialistischen Denkweisen, wie der antike Atomismus oder die modernen biologisch-mechanistischen Weltbilder, trotz ihrer durchaus bemerkenswerten Plausibilität etwas fade, und so ist der Mensch von jeher auf der Suche nach einer geeigneten Gewürzpalette.

\section{Dieser Moment, der in Erinnerung bleibt, wenn die therapeutische Geschäftigkeit und das Summen der Maschinen am Krankenbett verstummen.}

Im Laufe der Geschichte haben denn auch verschiedene Köche gemeint, eine solche gefunden zu haben, mit den bekannten, teilweise verheerenden Folgen für die jeweiligen Konsumenten.

\section{Stille Poesie}

Alle klinisch tätigen Ärzte und Ärztinnen haben in ihrem Berufsleben wiederholt Menschen beim Sterben begleitet. Es ist dieser jeweils ganz spezielle, eigentlich unspektakuläre Moment, der in Erinnerung bleibt, wenn die therapeutische Geschäftigkeit und das Summen der Maschinen am Krankenbett verstummen und einer weiten Ruhe, einer Art von Poesie Platz machen, die den Raum zu erfüllen scheint. Ein entspannter, friedvoller Augenblick der Besinnung, ein Aufscheinen gemeinsamer Geborgenheit, in der Patient und Arzt ganz einfach Mensch sind.

\section{Getön stürmischen Willens}

Das oftmals fast frohe, schon irgendwie der Zeit entrückte Aufleuchten in den Augen des Sterbenden, das je nach persönlichem Credo auch schon einer Vorschau ins Paradies oder alternativ der Wirkung von Endorphinen zugeschrieben wurde, findet in Arthur Schopenhauers (1788-1860) Hauptwerk, «Die Welt als Wille und Vorstellung», eine interessante philosophische Erläuterung. Den ausserempirischen Urgrund, die sogenannte metaphysische Ursache der Welt und aller ihrer Ereignisse, sieht dieser Denker in einem jenseits aller sinnlichen Erfassbarkeit und Sinnhaftigkeit stehenden, unbändigen Willen. Dieser kann nicht anders, als sich überall durchzusetzen, und so möchte er sich eben auch in der erfahrbaren Welt manifestieren. Dies geschieht in besonders intensivem Masse im Homo sapiens, der mit seinen Begehrlichkeiten und mit dem ihm eigenen, intelligenten, vorausplanenden und durchsetzungsmächtigen Zweckdenken ein exquisites Instrument für den blind und dumpf drängenden Willen darstellt. Dieser braucht also besonders uns, den Menschen, zu seiner konkreten, erfahrbaren Verwirklichung, und wir sind gleichzeitig ein Leben lang seine ruhelos getriebenen Sklaven. So kann der Tod und damit das Verlassen der Welt, die ja nichts anderes ist als die Manifestation des Willens, als echte Befreiung von dem letzteren verstanden werden. In diesem Sinne liessen sich auch die oftmals beobachteten entspannten Gesichtszüge des sterbenden Patienten erklären. Eine solche Loslösung von der Kraft des ungestümen Lebenswillens gibt es aber nur für den natürlichen Verlauf des Sterbens, wenn alle Teile des Körpers so ermüdet sind, dass sie allen Wünschen und Ansprüchen gewissermassen entsagen und sich nur noch nach Ruhe sehnen. Der Freitod hingegen ist für Schopenhauer kein echtes Entrinnen aus der Macht des Willens, im 
Gegenteil ist er gerade dessen kräftige Manifestation, weil der Selbstmord kein Dem-Leben-Entsagen ist, sondern vielmehr dem nicht erfüllten, verzweifelten Wunsch nach einer erträglicheren Lebenssituation entspringt. Diese Einsicht eines Philosophen, dessen Stärke öfters im Behaupten anstatt im Beweisen liegt,

\section{Eine solche Loslösung von der Kraft des ungestümen Lebenswillens gibt es aber nur für den natürlichen Verlauf des Sterbens.}

soll natürlich hinterfragt werden, ist aber trotzdem nicht uninteressant im Zusammenhang mit der Beihilfe zum Suizid, wo ja in letzter Zeit immer emsigere Protagonisten auftreten. Bei allem Respekt vor pragmatischen Entscheidungen bei tragischen Einzelfällen müssen wir Ärzte und Ärztinnen, bei der diesbezüglichen lauten Debatte um Pro und Kontra, doch stets bedenken, dass es zwar durchaus im Wesen unseres Berufes liegt, Patienten leider zu verlieren, aber eben trotz, und nicht kraft unserer Hilfe.

\section{Vokabular des ganz Anderen}

Die Beschäftigung mit dem Thema des Sterbens führte verschiedene Denker nicht nur zu Betrachtungen über den individuellen Tod, sondern auch zu Überlegungen über das Sein, das heisst über das innerste Wesen alles Weltlichen, über das, was dieses eigentlich ist, was oder wer es begründet oder gewissermassen hinter ihm steht. In diesem Zusammenhang kommt man, ob man nun will oder nicht, kaum an den fundamentalen Gedankengängen von Martin Heidegger (1889-1976) vorbei. So möchte ich diesen streitbaren Philosophen denn, obschon er hinsichtlich seiner Rolle im «Dritten Reich" von vielen Autoren scharf kritisiert wurde, doch kurz zu den obgenannten Fragen, um die Natur des Seins, zu Worte kommen lassen. Die traditionellen Antworten, die in diesem Zusammenhang bisher gegeben wurden, erschienen ihm als unbefriedigend, nicht weil sie allzu vage oder spekulativ gewesen wären, sondern weil sie allesamt das Sein prinzipiell falsch, nämlich als etwas Bestimmtes, zumindest als Denkkonzept Vorhandenes, verstanden haben. Ob Gottheit, Seele oder Bewusstsein, wie auch immer das Sein von seinen Vordenkern genannt wurde, stets handelte es sich um eine begrifflich definierte Entität, die dem Wesen des Seins nicht gerecht werden kann. Für Heidegger ist dieses das ganz Andere, weder sinnlich noch verstandesmässig als etwas Bestimmtes Erfassbare. Es ist keinesfalls ein besonders geartetes Ding, das anderes Dingliches bedingt, sondern etwas, das jenes ganz einfach nur sein, oder wie der Philosoph es auch formuliert, in sein Sein kommen lässt, das heisst zu etwas

\section{Poesie, welche imstande ist, auch Inhalte} aufzuzeigen, die ausserhalb jeder sprachlichen Logik liegen.

werden lässt, das «ist». Diese Idee eines Seins als blosses Seinlassen aller Dinge lässt sich mit unserer Sprache, die eine zumindest begriffliche Vorhandenheit ihrer Aussagen voraussetzt, gar nicht beschreiben, ja nicht einmal richtig denken, und wenn der Leser meine Schwierigkeiten teilt, Heidegger zu verstehen, dann ist das also absolut physiologisch. Aus diesem Grund sucht der Letztere denn auch verzweifelt nach einer Ausdrucksmöglichkeit, die seiner Seinsvorstellung gerecht werden könnte, und meint, diese schliesslich in der Poesie gefunden zu haben, welche imstande ist, auch Inhalte aufzuzeigen, die ausserhalb jeder sprachlichen Logik liegen. Sie braucht dazu nicht einmal unbedingt Buchstaben oder Laute, und damit schliesst sich auch der Kreis zu meinen anfänglichen Betrachtungen. Poesie kann sich nämlich in seltenen und ausserordentlichen Momenten auch einfach als stummes Bild ausdrücken. Auf diese Art vermag sie dann, normalsprachlich gar nicht Ausformulierbares zu vermitteln, so ähnlich vielleicht, wie wir es im Zimmer eines sterbenden Patienten erfahren können. 